\title{
Betatron radiation diagnostics for AWAKE Run 2
}

DOI:

10.1016/j.nima.2020.164076

\section{Document Version}

Accepted author manuscript

Link to publication record in Manchester Research Explorer

\section{Citation for published version (APA):}

Williamson, B., Xia, G., Gessner, S., Petrenko, A., Farmer, J., \& Pukhov, A. (2020). Betatron radiation diagnostics for AWAKE Run 2. Nuclear Instruments and Methods in Physics Research Section A: Accelerators,

Spectrometers, Detectors and Associated Equipment, 164076. https://doi.org/10.1016/j.nima.2020.164076

\section{Published in:}

Nuclear Instruments and Methods in Physics Research Section A: Accelerators, Spectrometers, Detectors and Associated Equipment

\section{Citing this paper}

Please note that where the full-text provided on Manchester Research Explorer is the Author Accepted Manuscript or Proof version this may differ from the final Published version. If citing, it is advised that you check and use the publisher's definitive version.

\section{General rights}

Copyright and moral rights for the publications made accessible in the Research Explorer are retained by the authors and/or other copyright owners and it is a condition of accessing publications that users recognise and abide by the legal requirements associated with these rights.

\section{Takedown policy}

If you believe that this document breaches copyright please refer to the University of Manchester's Takedown Procedures [http://man.ac.uk/04Y6Bo] or contact uml.scholarlycommunications@manchester.ac.uk providing relevant details, so we can investigate your claim.

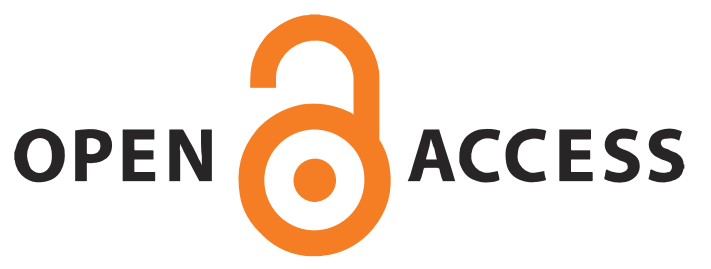




\section{Journal Pre-proof}

Betatron radiation diagnostics for AWAKE Run 2

B. Williamson, G. Xia, S. Gessner, A. Petrenko, J. Farmer, A. Pukhov

PII: $\quad$ S0168-9002(20)30501-5

DOI: $\quad$ https://doi.org/10.1016/j.nima.2020.164076

Reference: $\quad$ NIMA 164076

To appear in: $\quad$ Nuclear Inst. and Methods in Physics Research, A

Received date : 14 October 2019

Revised date: 7 February 2020

Accepted date : 29 April 2020

Please cite this article as: B. Williamson, G. Xia, S. Gessner et al., Betatron radiation diagnostics for AWAKE Run 2, Nuclear Inst. and Methods in Physics Research, A (2020), doi:

https://doi.org/10.1016/j.nima.2020.164076.

This is a PDF file of an article that has undergone enhancements after acceptance, such as the addition of a cover page and metadata, and formatting for readability, but it is not yet the definitive version of record. This version will undergo additional copyediting, typesetting and review before it is published in its final form, but we are providing this version to give early visibility of the article. Please note that, during the production process, errors may be discovered which could affect the content, and all legal disclaimers that apply to the journal pertain.

(C) 2020 Published by Elsevier B.V. 
Elsevier Editorial System(tm) for Nuclear

Inst. and Methods in Physics Research, A

Manuscript Draft

Manuscript Number: NIMA-D-19-01078R1

Title: Betatron radiation diagnostics for AWAKE Run 2

Article Type: Full length article

Section/Category: Accelerators, Beam Handling and Targets

Keywords: Accelerators; Beam driven plasma wakefield accelerators; beam diagnostics; betatron radiation

Corresponding Author: Mr. Barney Williamson, MPhys

Corresponding Author's Institution: University of Manchester

First Author: Barney Williamson, MPhys

Order of Authors: Barney Williamson, MPhys; Guoxing Xia; Spencer Gessner; Alexey Petrenko; John Farmer; Alexander Pukhov

Abstract: AWAKE Run 2 aims to preserve the transverse normalised emittance of an externally injected electron beam throughout acceleration, requiring a new diagnostic to measure the beam emittance after the accelerating plasma stage. Spectroscopy of the betatron emission from the electron beam could be suitable for this case. The method of trace-space reconstruction from a measured betatron spectrum is described via a simplified analytical model in order to assess its suitability the AWAKE experiment. The expected betatron emission from witness electrons at AWAKE is characterised using 3D quasi-static PIC simulations, revealing a measurable quantity of UV to soft x-ray emission. Practical challenges for the measurement are discussed. 


\section{Betatron radiation diagnostics for AWAKE Run 2}

B. Williamson ${ }^{\mathrm{a}, \mathrm{b}}$, G. Xia ${ }^{\mathrm{a}, \mathrm{b}}$, S. Gessner ${ }^{\mathrm{c}}$, A. Petrenko ${ }^{\mathrm{c}, \mathrm{d}}$, J. Farmer $^{\mathrm{f}, \mathrm{c}}$, A. Pukhov $^{\mathrm{e}}$

${ }^{a}$ The University of Manchester, Manchester, UK

${ }^{b}$ Cockcroft Institute, Warrington, UK

${ }^{c}$ CERN, Geneva, Switzerland

${ }^{d}$ Budker Institute of Nuclear Physics, Novosibirsk, Russia

${ }^{e}$ Heinrich Heine University, Düsseldorf, Germany

${ }^{f}$ Max-Planck-Institut für Physik, Munich, Germany

\section{Abstract}

AWAKE Run 2 aims to preserve the transverse normalised emittance of an externally injected electron beam throughout acceleration, requiring a new diagnostic to measure the beam emittance after the accelerating plasma stage. Spectroscopy of the betatron emission from the electron beam could be suitable for this case. The method of trace-space reconstruction from a measured betatron spectrum is described via a simplified analytical model in order to assess its suitability the AWAKE experiment. The expected betatron emission from witness electrons at AWAKE is characterised using 3D quasi-static PIC simulations, revealing a measurable quantity of UV to soft x-ray emission. Practical challenges for the measurement are discussed.

10 Keywords: Accelerators, Beam driven plasma accelerators, Beam diagnostics, Radiation

11 sources

\section{1. Introduction}

The AWAKE experiment [1] is a $\mathrm{GVm}^{-1}$-class plasma wakefield accelerator (PWFA) at 14 CERN. Electrons are injected into a 10-metre-long laser-ionised Rubidium plasma column from 15 an adjacent RF linac and accelerated in a wakefield driven by a $400 \mathrm{GeV}$ proton beam from the CERN SPS. A high-momentum proton driver facilitates large energy gains in a single stage of acceleration [2] and therefore reduces the complexity of a future collider based on plasma acceleration; other PWFA-based schemes, such as those driven with lepton beams, necessitate

* Corresponding author

Email address: barney.williamson@postgrad.manchester.ac.uk (B. Williamson) 
synchronisation and alignment between multiple plasma stages to overcome driver energy depletion and achieve beam energies useful for high energy physics experiments. Proof-of-concept experiments in AWAKE Run 1 have demonstrated electron acceleration in a proton-driven wakefield [3]. AWAKE Run 2 aims to preserve transverse electron beam emittance throughout acceleration and non-intercepting diagnostic systems to measure aspects of beam quality, such as the emittance of the accelerated electron beam, will therefore be central to the development of proton-driven PWFA. Betatron radiation has been shown to be a valuable single-shot, noninvasive diagnostic tool for laser-driven plasma wakefield accelerators (LWFAs): the average transverse beam size can be deduced from a measured critical frequency of betatron radiation and can also, when paired with an independent measurement of beam divergence and energy, be used to infer an uncorrelated normalised emittance [4-6]. Alternatively, the correlated emittance inside the plasma wakefield may be reconstructed with betatron and electron energy spectra alone, by analysing a betatron spectrum as the result of a convolution of single-particle orbits [7]. A diagnostic using the latter technique could be implemented for the AWAKE experiment in Run 2 , and here we set out the suitability of this method with some initial diagnostic considerations. In section 2 an approximate analytical model of the betatron radiation from a generic beam of electrons is introduced to demonstrate how the correlated beam emittance is recovered from a betatron spectrum. Section 3 presents particle-in-cell simulations that estimate the quantity and properties of the betatron radiation forseen in AWAKE Run 2. The effect of a varying transverse beam size on the betatron emission is shown, and requirements for the use of the reconstruction technique are described. Practical considerations for implementing a diagnostic system based on betatron radiation spectroscopy are described in section 4 , and the number of detectable photons is estimated using the simulated emission.

\section{Betatron Radiation}

Electrons in ion cavities execute transverse, or betatron, oscillations and emit radiation with spectral characteristics determined by the distribution of single-electron orbits within a beam envelope [8]. With constant energy, and in the absence of radiation reaction, transverse electron motion in a fully blown-out ion channel within a plasma of electron density $n_{p}$ is governed by the focusing force from the resulting wakefield. It works against the transverse electron momentum to produce sinusoidal betatron oscillations with a frequency $\omega_{\beta}=\omega_{p} / \sqrt{2 \gamma}$ and 
49 a period $\lambda_{\beta}=2 \pi / k_{\beta} \simeq 2 \pi \sqrt{2 \gamma} / k_{p}$, where $\omega_{p}=\left(n_{p} e^{2} / m_{e} \epsilon_{0}\right)^{1 / 2}$ and $k_{p}$ is the wavenumber

50 associated with the plasma wavelength. The electron Lorentz factor is $\gamma$ and $m_{e}, e$ and $\epsilon_{0}$ are the

51 electron mass, electron charge and vacuum permittivity respectively.
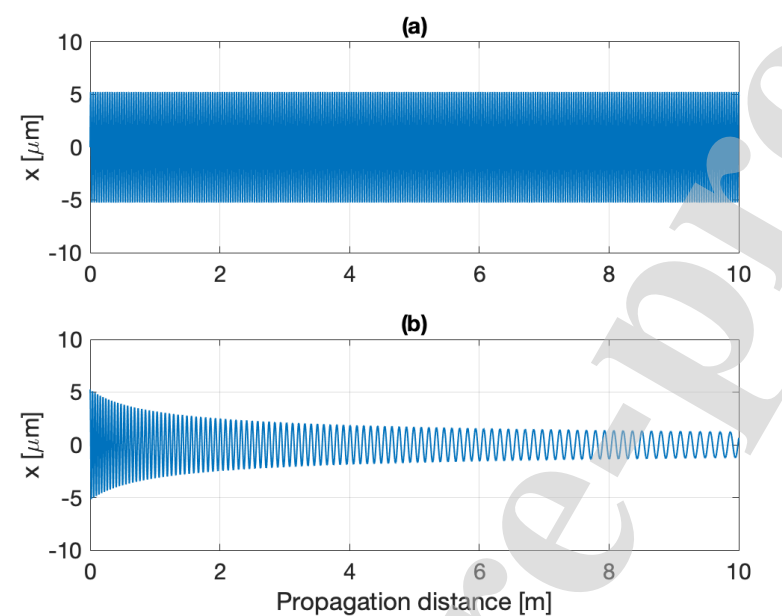

Figure 1: The transverse motion of an electron trapped in an ion channel, both in the case of constant energy (a) and with a non-zero accelerating field $E_{z}(\mathrm{~b})$.

If the electron energy varies, e.g. in the case of a non-zero accelerating field $E_{z}$, the equation

53 of motion can instead be modelled as a parametric oscillator of slowly varying frequency. This

54 results in a decaying amplitude and frequency of betatron motion as an electron gains energy,

55 known as adiabatic damping. To illustrate this in the case of an electron accelerated at AWAKE,

56 figure 1 shows the transverse motion of a single electron (initially with $\gamma_{0}=426$ ), trapped in an

57 ion channel with $n_{p}=7 \times 10^{14} \mathrm{~cm}^{-3}$, for both constant energy and with a non-zero accelerating

58 field $E_{z}=0.14 E_{0}$ where $E_{0}=m_{e} c \omega_{p} / e$ is the wave-breaking field and $c$ the speed of light in

59 vacuum. These parameters correspond to the baseline case for AWAKE Run 2. The strength

${ }_{60}$ of transverse oscillation is characterised by the parameter $\alpha_{\beta}=\gamma k_{\beta} r_{\beta}$, where $r_{\beta}$ is the betatron

${ }_{61}$ amplitude. The ensemble average $\overline{r_{\beta}}$ is equivalent to the Gaussian beam radius $\sigma_{r}$. For $\alpha_{\beta}>>$

621 the electron motion results in a broadband spectrum that is synchrotron-like, emitted within

${ }_{63}$ an angle $\theta \sim \alpha_{\beta} / \gamma$ from the acceleration axis, and has a shape given by the universal function

${ }_{64} S\left(\omega / \omega_{c}\right):$

$$
S(x)=x \int_{x_{3}}^{\infty} K_{5 / 3}(\xi) d \xi,
$$


where $K_{5 / 3}$ is a modified Bessel function of the second kind. Such a spectrum falls expo66 nentially after a critical frequency $\omega_{c}$, which divides the spectrum into two equal parts and, for a 67 relativistic electron in a pure ion column, may be expressed [9]

$$
\omega_{c}=\frac{3}{2} \gamma^{3} c r_{\beta} k_{\beta}^{2} .
$$

The power radiated by an electron over all angles, averaged over one betatron period, is [10]

$$
\left\langle P_{\gamma}\right\rangle=\frac{\pi e^{2} c}{3 \epsilon_{0}} \frac{\gamma^{2} \alpha_{\beta}^{2}}{\lambda_{\beta}}=\frac{\pi e^{2} c}{3 \epsilon_{0}} \frac{\gamma^{4} k_{\beta}^{2} r_{\beta}^{2}}{\lambda_{\beta}} .
$$

${ }_{88} W_{j}$ is found according to equation (5) as seen in 2(d).

where $\alpha$ is the fine structure constant.

\subsection{Emittance Reconstruction}

Using equations (1-4) a simple radiation model can be created. We start with a plasma of density $n_{p}=1 \times 10^{18} \mathrm{~cm}^{-3}$, within which a radially symmetric Gaussian electron beam of $\sigma_{r}=$ $1.325 \mu \mathrm{m}$ propagates for one betatron oscillation in a fully blown-out ion channel. The radiating beam has $\gamma=2000$ and contains $1 \mathrm{pC}$ of charge $\left(6.25 \times 10^{6}\right.$ electrons $)$. We assume zero energy spread $\sigma_{\gamma}=0$, that $\sigma_{r} \simeq r_{\beta, R M S}$ and $\alpha_{\beta}>>1$ for most $r_{\beta, i}$. The ion channel is generated by a separate drive beam, itself with a density $n_{d}$ such that $n_{d} / n_{p}>>1$. With equation (1) a series of synchrotron spectra $S\left(\omega_{j} / \omega_{c, i}\right)$ are generated, covering a range of frequencies $\omega_{j}$ and betatron amplitudes $r_{\beta, i}$, which each have a corresponding critical frequency $\omega_{c, i}$ given by equation (2). The resulting spectral distribution is shown in figure 2(a). Each spectrum $S\left(\omega / \omega_{c, i}\right)$ is then assigned a number photons, according to its critical energy $\omega_{c, i}$, using equation (4), as shown in figure 2(b). This spectral distribution $W_{i, j}$ represents the number of photons emitted per electron, in one betatron oscillation, over a range of $i$ betatron amplitudes, and $j$ photon energies $\left(\hbar \omega_{j}\right)$. A Gaussian distribution of betatron amplitudes $R\left(r_{\beta, i}\right)$ with $r_{\beta, R M S}=1.325 \mu \mathrm{m}$ is used to populate $W_{i, j}$ and is shown in 2(c). Finally, when summed over all betatron amplitudes $r_{\beta, i}$, a total spectrum 

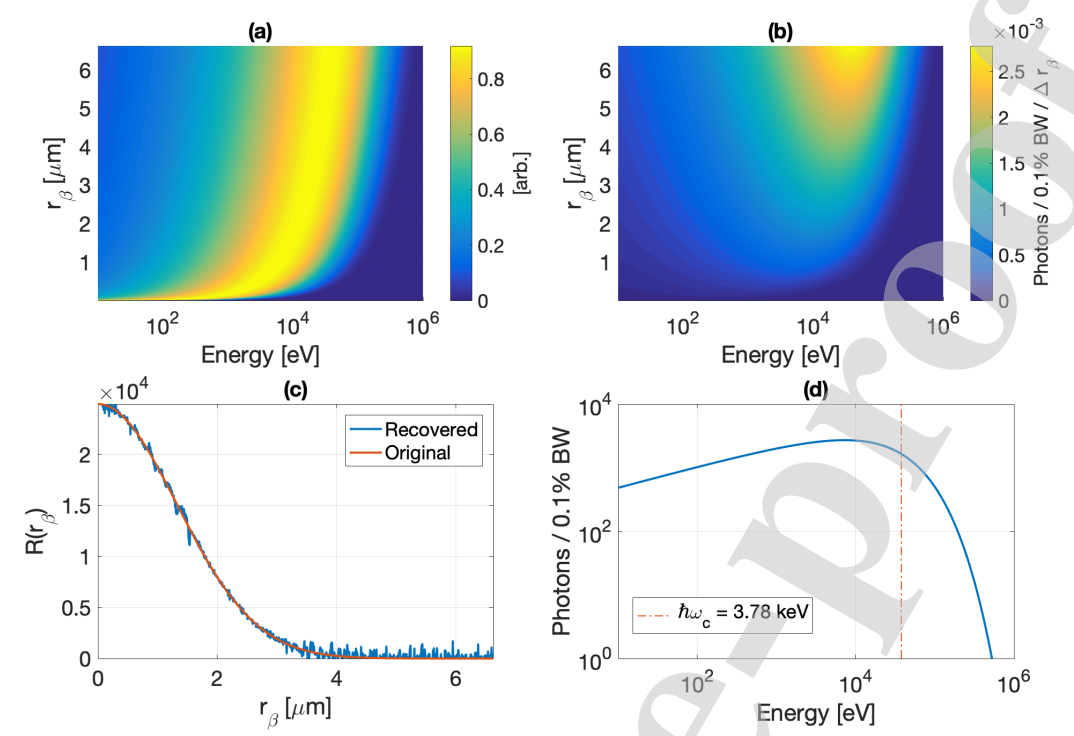

Figure 2: (a) The spectral distribution $S\left(\omega_{j} / \omega_{c, i}\right)$; (b) the spectral distribution emitted per electron $W_{i, j}$, where $\Delta \hbar \omega=$ $10^{-3} \hbar \omega$ and $\Delta r_{\beta}=10^{-3} r_{\beta}$; (c) the original and recovered Gaussian beam distribution $R\left(r_{\beta, i}\right)$; and (d) the total spectrum $W_{j}$, the result of populating $W_{i, j}$ and summing over all betatron amplitudes, which has a critical energy $\hbar \omega_{c}=3.78 \mathrm{keV}$ (dash-dotted line).

$$
W_{j}=\sum_{i} R\left(r_{\beta, i}\right) W_{i, j}
$$

$$
R\left(r_{\beta, i}\right)=\sum_{j} \Sigma_{j} W_{i, j}^{-1},
$$

enables the reconstruction of a beam profile $R$ from a measured spectrum $\Sigma_{j}$, given an inverse matrix $W_{i, j}^{-1}$, where $W_{i, j}^{-1}$ is the Moore-Penrose pseudoinversion of the radiation model $W_{i, j}$. By considering the modified Lorentz factor due to the relativistic transverse motion, it is possible to approximate the divergence $\theta$ due to the betatron motion itself [7]: 


$$
\theta=\sqrt{\frac{\sqrt{1+\frac{1}{2} \gamma^{2} r_{\beta}^{2} k_{\beta}^{2}}}{4 \gamma}} r_{\beta} k_{p} .
$$

With a series of weighted positions $x_{i}=R\left(r_{\beta, i}\right)$, and corresponding angles $x_{i}^{\prime}=\theta\left(r_{\beta, i}\right)$, the correlated geometric r.m.s. emittance may be calculated via equation (8), where angle brackets represent the average over $i$ values.

$$
\varepsilon_{r . m . s .}=\sqrt{\left\langle x^{2}\right\rangle\left\langle x^{\prime 2}\right\rangle-\left\langle x x^{\prime}\right\rangle^{2}} .
$$

Testing this procedure by setting $\Sigma_{j}=W_{j}$ recovers a beam with a transverse profile (also shown in figure 2(c)) which, if it had emitted the spectrum shown in 2(d), would have a geometric emittance $\varepsilon_{\text {r.m.s. }}=2.37 \times 10^{-3} \mathrm{~mm} \mathrm{mrad}$ and normalised emittance $\varepsilon_{n} \simeq \gamma \varepsilon_{\text {r.m.s. }}=4.74 \mathrm{~mm} \mathrm{mrad}$. Such a model could be used to reconstruct the transverse normalised emittance of any independently measured betatron spectrum $\Sigma_{j}$ so long as the assumptions of the model are fulfilled, i.e. electrons emit in wiggler motion over one betatron period.

\section{Simulated Betatron Emission}

The three-dimensional quasi-static particle-in-cell (PIC) code QV3D [11], built on the framework of VLPL [12], is used in the presented simulation study. PIC simulations do not explicitly model synchrotron-like emission, as the short wavelengths cannot be resolved by the simulation grid. In QV3D, the emission is calculated analytically per particle, per timestep. Each macroparticle emits a synchrotron spectrum with a shape given by equation (1) along its trajectory, from which the transverse momentum change of the macroparticle in one timestep is calculated. This change in momentum determines how many photons of that spectrum are emitted. The emission (critical frequency, photon count and direction in polar angle $\theta$ and azimuthal angle $\phi$ ) is saved, and the macroparticle momentum updated, which also allows radiation reaction to be modelled [13]. The raw spectrum from the code is saved as a critical photon spectrum $N\left(\omega_{c}\right)$; the total betatron spectrum $N(\omega)$ is reconstructed by convolving equation (1) with $N\left(\omega_{c}\right)$ :

$$
N(\omega)=\int_{0}^{\infty} S\left(\frac{\omega}{\omega_{c}}\right) N\left(\omega_{c}\right) d \omega_{c} .
$$




\subsection{AWAKE Run 2}

In AWAKE Run 2, the two-dimensional transverse normalised emittance $\varepsilon_{n}^{*}$ will be preserved at its injected value by matching the electron beam to the plasma, while the final relative energy spread $\sigma_{\gamma_{f}} /\left\langle\gamma_{f}\right\rangle$ will be kept to the \%-level by beamloading the proton-driven wakefield. Beam parameters to meet these conditions were previously determined by Olsen et al. (2018) [14]. Matching requires an initial equilibrium transverse beam size that, for a given beam energy, will result in a preserved normalised emittance [15]. The electron beam drives its own local nonlinear plasma wave in addition to the proton-driven wakefield in order to facilitate matching. The transverse dynamics of the electron beam are then somewhat independent from the quasi-linear wakefield driven by the proton beam, whose principle role is to provide longitudinal acceleration. In this section we reproduce the Run 2 model of Olsen et al. [14] and include betatron radiation emission in order to provide estimates for the number of detectable photons for a betatron radiation diagnostic at AWAKE. Simulations in QV3D were performed with cell sizes of $\Delta x=\Delta y=0.01 c / \omega_{p}$ and $\Delta z=0.1 c / \omega_{p}$ in the transverse and longitudinal directions respectively. Four particles per cell were used for the bulk plasma, which had an electron density $n_{p}=$ $7 \times 10^{14} \mathrm{~cm}^{-3}$. The simulation domain had dimensions of $10 \times 5 \times 5 \mathrm{c} / \omega_{p}$. To avoid simulation of the full proton beam a single short bunch, with transverse size $\sigma_{x, y}=200 \mu \mathrm{m}, \sigma_{z}=40 \mu \mathrm{m}$ that contains $1.46 \times 10^{10}$ protons $\left(n_{p b}=0.83 n_{p}\right)$, is used to produce the accelerating field $E_{z}$. The proton beam energy was set with $\gamma_{p b}=426$, the typical value on extraction from the CERN SPS. In the simulation the proton mass is increased by a factor of $10^{6}$ in order to suppress the transverse evolution of the proton beam, and therefore eliminate any betatron radiation it might produce. The witness electron beam has $\gamma_{e b}=426, \sigma_{x, y}=5.25 \mu \mathrm{m}, \sigma_{z}=60 \mu \mathrm{m}$ and contains $100 \mathrm{pC}\left(6.25 \times 10^{8}\right.$ electrons $)$. With $n_{e b}=34.3 n_{p}$ the electron beam is well within the blow-out regime. The initial $\varepsilon_{n}^{*}$ and energy spread $\sigma_{\gamma_{i}} /\left\langle\gamma_{i}\right\rangle$ are $2 \mathrm{~mm} \mathrm{mrad}$ and $0.1 \%$ respectively. In Figure 3 (a) the simulated emission integrated over all angles is shown with $\hbar \omega_{c}=357 \mathrm{eV}$, containing a total of $N_{e}=9.83 \times 10^{8}$ photons. Figures 3 (b-d) show the evolving beam parameters: energy spread saturates at less than $6 \%$, while the normalised emittance remains close to the matched value; some emittance growth is seen, due largely to the erosion of the bunch head by interaction with the plasma. The angular betatron spectrum for $\theta>1 \mathrm{mrad}$ from the acceleration axis $z$ is shown in figure 4. In this baseline AWAKE Run 2 case, with $\sigma_{x, y}=5.25 \mu \mathrm{m}$, most emitted photons $(96.9 \%$ ) are confined to small angles less than $1 \mathrm{mrad}$ and have energies in the 

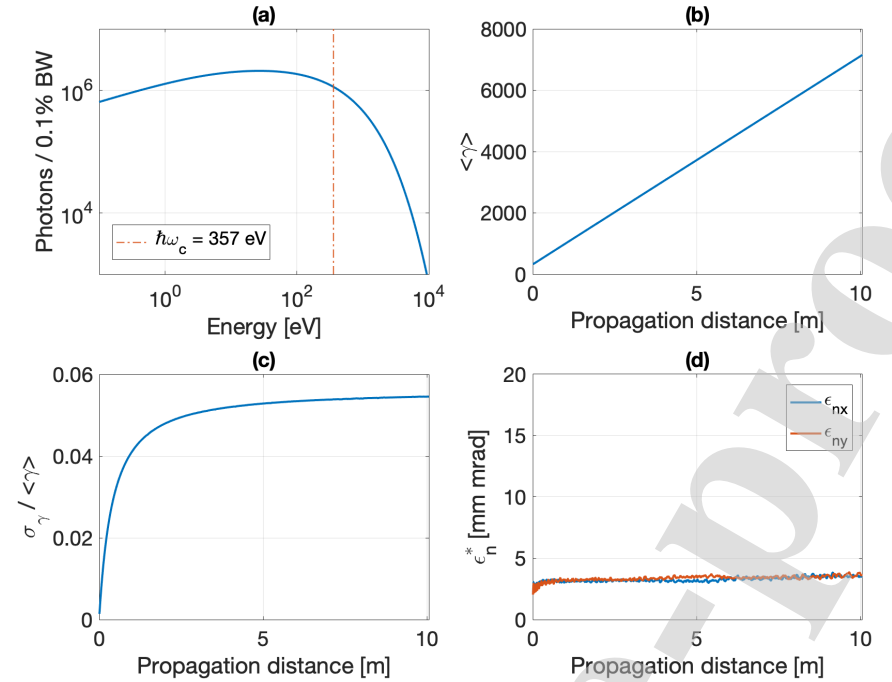

Figure 3: The angle-integrated $(0<\theta<5 \mathrm{mrad})$ betatron spectrum $N(\omega)$ (a) and electron beam $\gamma$, relative energy spread $\sigma_{\gamma} /\langle\gamma\rangle$ and transverse normalised emittance $\varepsilon_{n}^{*}$ over 10 metres (b-d) for the baseline AWAKE Run 2 case with $\sigma_{x, y}=$ $5.25 \mu \mathrm{m}$. The spectrum gives the number of photons in $0.1 \%$ bandwidth $\left(\Delta \hbar \omega=10^{-3} \hbar \omega\right)$ per photon energy $\hbar \omega$.

range $1-1000 \mathrm{eV}$. Above $1 \mathrm{mrad} 3.1 \%$ remain which, although a small fraction of the total, still provides a reasonable number of photons per $0.1 \% \mathrm{BW}$ in absolute terms. These photons have lower energies in the range $1-100 \mathrm{eV}$. To determine the effect of larger beam radii on the emitted betatron spectrum, the baseline simulation was scanned between $\sigma_{x, y}=5.25 \mu \mathrm{m}$ and $\sigma_{x, y}=$ $40 \mu \mathrm{m}$. The resulting angle-integrated spectra are shown in figure 5(a). According to equation (2), $\omega_{c}$ should scale linearly with $r_{\beta}$ and equivalently with $\sigma_{x, y}$. However, the simulated spectra show only small changes in the critical energy $\hbar \omega_{c}$ over a range of different beam sizes. Two mechanisms limit the energy of emitted photons to produce the simulated spectra: firstly, due to adiabatic damping of $\sigma_{x, y}$ discussed in section 1 , the beam sizes fall to less than $5 \mu \mathrm{m}$ in all cases. Secondly, an increasing electron beam size results in a falling beam density $n_{e b}$ and ratio $n_{e b} / n_{p}$ which, since the electron beam blows out its own ion channel, results in an increasingly linear wakefield with weaker transverse forces to drive the betatron motion. With $\sigma_{x, y}=40 \mu \mathrm{m}$ the ratio falls to $n_{e b} / n_{p}=0.59$, and $\hbar \omega_{c}$ is lower than the emission from the smaller $\sigma_{x, y}=20 \mu \mathrm{m}$ beam. Figure 5(b) shows that neither the number nor divergence of photons emitted into angles $\theta>1 \mathrm{mrad}$ have a strong scaling with transverse beam size, although they do increase. 


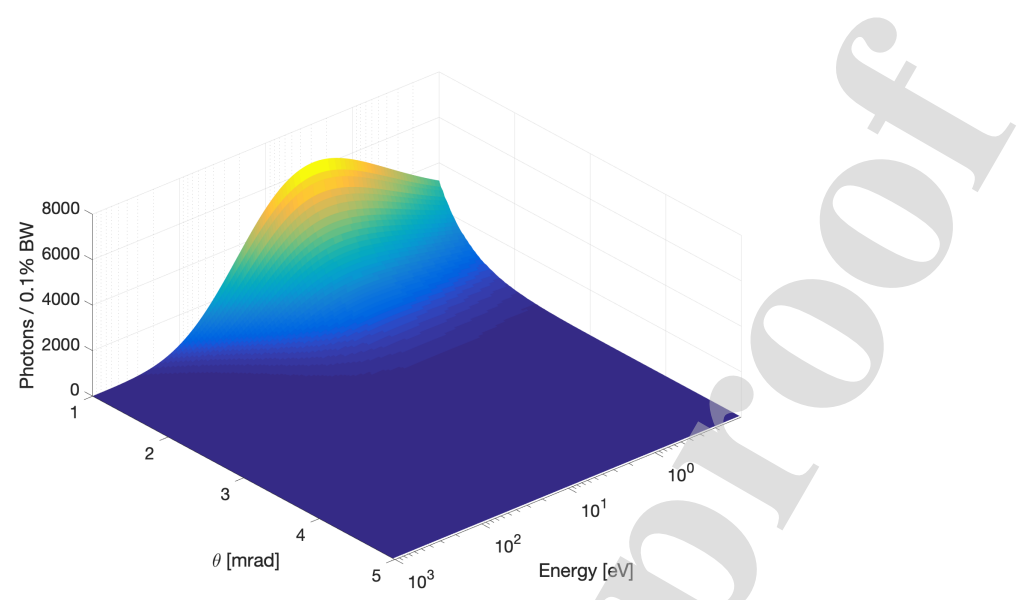

Figure 4: The total spectrum $N(\omega, \theta)$ distributed in polar angle $\theta>1 \mathrm{mrad}$ for the baseline AWAKE Run 2 case with $\sigma_{x, y}=5.25 \mu \mathrm{m}$. This angular spectrum gives the number of photons in $0.1 \%$ bandwidth $\left(\Delta \hbar \omega=10^{-3} \hbar \omega\right)$ per photon energy $\hbar \omega$ per polar observation angle $\theta$
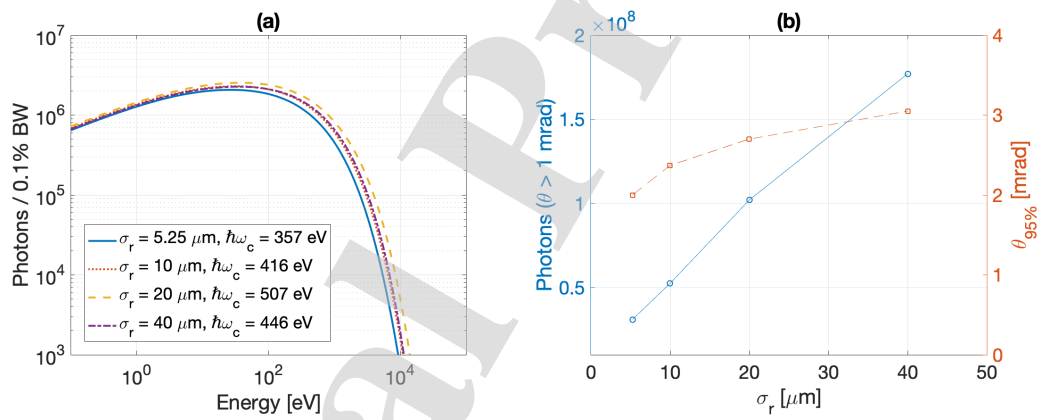

Figure 5: The angle-integrated $(0<\theta<5 \mathrm{mrad})$ spectra $N(\omega)$ for the AWAKE Run 2 baseline case scanned over beam size, from $\sigma_{x, y}=5.25 \mu \mathrm{m}$ to $\sigma_{x, y}=40 \mu \mathrm{m}$ (a). The spectrum gives the number of photons in $0.1 \%$ bandwidth ( $\Delta \hbar \omega=10^{-3} \hbar \omega$ ) per photon energy $\hbar \omega$. The number of photons emitted for $\theta>1 \mathrm{mrad}$ (solid line) and the radiation divergence $\theta_{95 \%}$ as a function of beam size $\sigma_{x, y}$ (dashed line) is shown in (b). $\theta_{95} \%$ is defined as the angle that contains $95 \%$ of the photons remaining above 1 mrad.

The one-dimensional beam profile cannot be reconstructed from these simulated spectra using the simple analytical model presented in section 2. This is because the model does not account for evolving beam properties, and therefore cannot simulate betatron emission when it is integrated over multiple betatron periods. At AWAKE the electron beam will complete $\sim 100$ betatron periods along the accelerator and the beam size and energy will continually evolve during this time. The combination of a relatively modest accelerating gradient and many betatron 
oscillations means that the radiating electron beam contributes a significant portion of the final betatron spectrum well before it has reached its final energy, despite the strong scaling of radiated power with $\gamma$ seen in equation (3). Therefore for the experimental measurement itself, a radiation model that accounts for beam evolution must be employed. This is an ongoing area of study. Electron orbits that correspond to undulator motion, i.e. $\alpha_{\beta}<<1$, should also be considered; for an AWAKE-like beam with $\sigma_{r}=5.25 \mu \mathrm{m}$ in an ion channel with $n_{p}=7 \times 10^{14} \mathrm{~cm}^{-3}$, a large fraction of the electrons in the $1 \sigma$ beam core oscillate with $\alpha_{\beta}<1$. Consequently, a singleparticle model [8] that reproduces the spectrum under all regimes of electron motion should be implemented in order to accurately reconstruct the beam profile [7]. Furthermore, the electron beam energy spread following acceleration will have an appreciable impact on the final betatron spectrum, and the beam profile and emittance reconstruction require an independent electron beam energy spectrum.

\section{Diagnostic Considerations}

Betatron emission from LWFAs has been measured using a number of techniques. It can be measured directly after its parent beam has been removed [4], but may also be extracted and measured indirectly via a highly oriented pyrolytic graphite (HOPG) crystal [16]. The spectrum can be reconstructed with an x-ray charge-coupled device (CCD) in single-photon counting mode, or with a set of Ross filters. Betatron radiation from a PWFA has also been measured previously [17]. The AWAKE experiment is a challenging environment for measuring betatron radiation as a substantial x-ray background exists from upstream interaction of SPS protons with the beamline, and because the relativistic proton beam itself is rigid and cannot be transversely kicked to isolate the betatron radiation. Core and defocused protons have a divergence of $1 \mathrm{mrad}$ on exiting the plasma and their presence inhibits a direct, on-axis measurement. However, as shown in figure 4, a significant remnant of the emission falls outside $1 \mathrm{mrad}$. A measurement station $5 \mathrm{~m}$ downstream from the accelerating plasma stage with a hole radius corresponding to such an angle, e.g. $5 \mathrm{~mm}$ radius at $5 \mathrm{~m}$, would allow the proton beam to pass and enable a partial measurement of the emission, i.e. those photons having energies between $1-100 \mathrm{eV}$ and falling in angles above $1 \mathrm{mrad}$.

A conceptual layout is show in figure 6. A combination of conventional $\mathrm{Al}$ and multilayered mirrors are readily available to measure in the $1-100 \mathrm{eV}$ range. Multilayered optics could be 


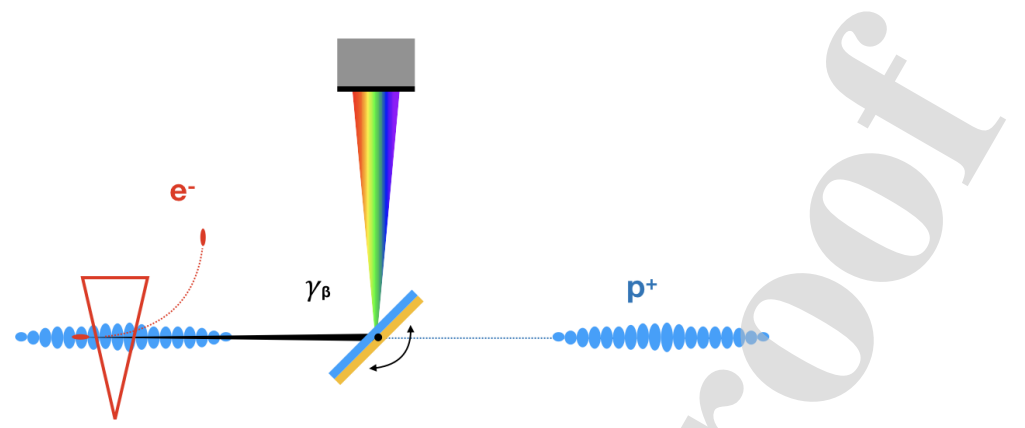

Figure 6: Layout for a potential betatron radiation diagnostic for AWAKE Run 2. Electrons $e^{-}$are kicked by the energy spectrometer dipole, leaving protons $p^{+}$and betatron radiation $\gamma_{\beta}$ to propagate. An array of on-axis UV-coated Al or multilayered mirrors may be used to pick off betatron photons in the $1-100 \mathrm{eV}$ range, to be detected by a UV-VUV CCD camera.

used to select certain photon energies or, if rotated according to Bragg's law, be used to build a spectrum. Ultraviolet (UV) photons with energies $\geq 3.1 \mathrm{eV}$ cannot propagate in air and require vacuum, further complicating the measurement. The measurement would profit from being split into two phases. First a UV measurement between $200-400 \mathrm{~nm}$ wavelengths $(<3.1 \mathrm{eV})$ can be used to extrapolate a spectrum and estimate the beam size $\sigma_{r}$, and would confirm the observation of a significant quantity of betatron radiation. Second, a vacuum UV (VUV) measurement $(3.1-100 \mathrm{eV})$ that provides spectroscopy in a larger range, improving the precision of the fitted spectrum and enabling correlated emittance reconstruction. A local background of transition radiation from strongly defocused protons ( $>1 \mathrm{mrad}$ ) striking the UV/VUV optics would reduce the signal to noise ratio on each shot. However this could be addressed by characterising the signal in the absence of accelerated electrons. Finally, due to the x-ray background in the beamline, sensitive cameras should be positioned at some minimum distance from the beamline, which could further reduce the number of detectable photons. Using the photon emission from the baseline simulation $\left(\sigma_{x, y}=5.25 \mu \mathrm{m}\right)$ in the previous section it is possible to estimate the number of detectable photons per unit area of sensor $N_{d}$ from the total emitted, $N_{e}$ :

$$
N_{d}=g R_{m} \delta_{\theta} N_{e}
$$

where $\delta_{\theta}=0.031$ is the fraction of photons falling above $1 \mathrm{mrad}$, determined in the simulations, and $R_{m}$ is the fraction reflected by the mirror, assumed to be 0.6 . The geometrical factor 
$g=\delta \Omega / 4 \pi$ accounts for the loss of photons over a distance $r$ between the mirror surface and the CCD sensor plane. The sensor with area $A$ occupies a solid angle $\delta \Omega=A / r^{2}$. A VUV CCD camera a such as an Andor Newton SO has a sensor area of $178.89 \mathrm{~mm}^{2}$, and a $26 \mu \mathrm{m} \times 26 \mu \mathrm{m}$ pixel size. From the baseline simulation $N_{e}=9.83 \times 10^{8}$ photons, this camera positioned $200 \mathrm{~mm}$ from the reflecting mirror should be subject to $6.62 \times 10^{3}$ photons $\mathrm{mm}^{-2}$. With the native pixel area of $6.76 \times 10^{-4} \mathrm{~mm}^{2}$ this may be too few photons to register above the noise floor of the CCD sensor. However, with $8 \times 8$ pixel binning, the pixel area may be increased to $4.33 \times 10^{-2} \mathrm{~mm}^{2}$ and the photons per pixel increased from $\sim 5$ to $\sim 286$. Finally, assuming the CCD QE $\sim 0.6$ one could expect $\sim 172$ detected photons per binned pixel in the range 1-100 eV. Pixel binning would result in a poorer sensor resolution of $\sim 200 \mu \mathrm{m}$, although at $5 \mathrm{~m}$ downstream from the accelerator, the radiation divergence of $\theta=2 \mathrm{mrad}$ for the baseline case, shown in figure 5(b), would produce a relatively large $10 \mathrm{~mm}$ spot radius. Despite the weak scaling of radiation properties with $\sigma_{x, y}$ seen in simulations, larger beams sizes would still produce a broader distribution containing more photons, which would increase the number of detectable photons. Only $28 \%$ of the total detectable photons have an energy $<3.1 \mathrm{eV}$ in the baseline case, so a larger sensor may be required for the intial lower energy measurement.

\section{Conclusions}

The next phase of the AWAKE experiment aims to demonstrate the preservation of transverse beam emittance throughout acceleration. This requires a new emittance measurement after the accelerating plasma stage, which could be facilitated by betatron radiation spectroscopy in conjunction with the electron beam energy spectrum. The reconstruction of the one-dimensional electron beam transverse profile and emittance is possible with a simple betatron radiation model in the limit of full wiggler motion. However, owing to both the adiabatic damping of the electron beam size over many betatron periods and quasi-undulator motion of electrons accelerated at AWAKE, a diagnostic reconstructing the beam emittance will require a single-particle betatron radiation model that takes account of the evolving beam properties over the $10 \mathrm{~m}$ plasma column. This is currently under development. The angular acceptance of the measurement is limited by the diverging proton beam, however quasi-static three-dimensional simulations show that a reasonable quantity of soft $\mathrm{x}$-ray to UV betatron photons will be produced in AWAKE Run 2 that will be measurable above $1 \mathrm{mrad}$ and away from the proton beam. The number and spatial 
distribution of photons will be relatively insensitive to the electron beam transverse size as the beam drives its own wakefield, which becomes increasingly linear and provides weaker focusing as the beam density falls. Due to the restrictions of the experimental environment UV-VUV (1-100 eV) spectroscopy could be the most promising method to partially measure the betatron spectrum and reconstruct the emittance of accelerated electrons, however issues such as camera positioning and background radiation suppression must be addressed.

\section{Acknowledgements}

This work was supported by the Science and Technologies Facilities Council (STFC) grant number ST/N504129/1, and A. Pukhov aknowledges support by the German Research Foundation (DFG). Computing resources provided by the CERN High Performance Computing service. The Authors would like to thank Alessandro Curcio for helpful discussions.

\section{References}

[1] E. Gschwendtner, E. Adli, L. Amorim, et al., AWAKE: A Proton-Driven Plasma Wakefield Acceleration Experiment at CERN, Nucl. Instruments Methods Phys. Res. Sect. A 829 (2016) 76-82.

[2] A. Caldwell, K. Lotov, A. Pukhov, F. Simon, Proton-driven plasma-wakefield acceleration, Nature Physics 5 (5) (2009) 363-367.

[3] E. Adli, et al. (AWAKE Collaboration), Acceleration of electrons in the plasma wakefield of a proton bunch, Nature 561 (7723) (2018) 363-367.

[4] G. R. Plateau, C. G. Geddes, D. B. Thorn, et al., Low-emittance electron bunches from a laser-plasma accelerator measured using single-shot X-ray spectroscopy, AIP Conf. Proc. 1507 (June) (2012) 278-283.

[5] M. Schnell, A. Sävert, B. Landgraf, et al., Deducing the electron-beam diameter in a laser-plasma accelerator using X-ray betatron radiation, Phys. Rev. Lett. 108 (7) (2012) 075001(5).

[6] A. Köhler, J. P. Couperus, O. Zarini, et al., Single-shot betatron source size measurement from a laser-wakefield accelerator, Nucl. Instruments Methods Phys. Res. Sect. A 829 (2016) 265-269.

[7] A. Curcio, M. Anania, F. Bisesto, et al., Trace-space reconstruction of low-emittance electron beams through betatron radiation in laser-plasma accelerators, Phys. Rev. Accel. Beams 20 (1) (2017) 012801(8).

[8] E. Esarey, B. A. Shadwick, P. Catravas, W. P. Leemans, Synchrotron radiation from electron beams in plasmafocusing channels, Phys. Rev. E 65 (5) (2002) 056505(15).

[9] I. Kostyukov, S. Kiselev, A. Pukhov, X-ray generation in an ion channel, Phys. Plasmas 10 (12) (2003) $4818-4828$.

[10] S. Corde, K. Ta Phuoc, G. Lambert, R. Fitour, V. Malka, A. Rousse, A. Beck, E. Lefebvre, Femtosecond x rays from laser-plasma accelerators, Rev. Mod. Phys. 85 (1) (2013) 1-48.

[11] A. Pukhov, Particle-in-Cell Codes for plasma-based particle acceleration, Cern Yellow Reports 1 (2016) 181-206. 
[12] A. Pukhov, Three-dimensional electromagnetic relativistic particle-in-cell code vlpl (virtual laser plasma lab), Journal of Plasma Physics 61 (3) (1999) 425-433.

[13] J. P. Farmer, A. Pukhov, Tev acceleration in a matryoshka plasma channel (2019). arXiv:1910.07488.

[14] V. K. Olsen, E. Adli, P. Muggli, Emittance preservation of an electron beam in a loaded quasilinear plasma wakefield, Phys. Rev. Accel. Beams 21 (1) (2018) 011301(8).

[15] E. Esarey, P. Sprangle, J. Krall, A. Ting, Overview of plasma-based accelerator concepts, IEEE Transactions on Plasma Science 24 (2) (1996) 252-288.

[16] M. Šmíd, I. Gallardo González, H. Ekerfelt, J. Björklund Svensson, M. Hansson, J. C. Wood, A. Persson, S. P. Mangles, O. Lundh, K. Falk, Highly efficient angularly resolving x-ray spectrometer optimized for absorption measurements with collimated sources, Rev. Sci. Instrum. 88 (6) (2017) 063102(8).

[17] S. Wang, C. E. Clayton, B. E. Blue, et al., X-Ray Emission from Betatron Motion in a Plasma Wiggler, Phys. Rev. Lett. 88 (13) (2002) 135004(4). 
- AWAKE Run 2 aims to demonstrate the preservation of beam quality throughout acceleration

- New diagnostics are required to measure transverse emittance of the accelerated electron beam

- This article proposes using UV-VUV spectroscopy to reconstruct the electron beam emittance, and the reconstruction technique is described

- Quasi-static three-dimensional simulations with the particle-in-cell code QV3D are used to provide estimates for the radiation spectrum and spatial distribution for expected electron beam properties in AWAKE Run 2

- The number of detectable photons is estimated in order to inform the measurement 
Barney Williamson: Writing - Original Draft, Writing - Review \& Editing, Methodology, Software, Formal Analysis, Visualization, Investigation. Guoxing Xia: Supervision, Funding acquisition, Resources. Spencer Gessner: Conceptualization, Supervision, Resources. Alexey Petrenko: Software, Supervision, Resources, Methodology. John Farmer: Software, Supervision, Writing Review \& Editing. Alexander Pukhov: Software, Supervision, Resources. 


\section{*Declaration of Interest Statement}

\section{Declaration of interests}

VThe authors declare that they have no known competing financial interests or personal relationships that could have appeared to influence the work reported in this paper.

The authors declare the following financial interests/personal relationships which may be considered as potential competing interests:

None 